\title{
Analysis of Random Access Protocol under Bursty Traffic
}

\author{
Jianbo Gao and Izhak Rubin \\ Electrical Engineering Department, University of California, \\ Los Angeles, CA 90095 \\ $\{$ jbgao, rubin\}@ee.ucla.edu
}

\begin{abstract}
Aloha-type random-access protocols have been employed as access control protocols in wireline and wireless, stationary and mobile, multiple-access communications networks. They are frequently employed by the control and signaling subsystem of demand-assigned multiple access protocols for regulating the sharing of a control channel. The latter is used for the transport of reservation packets requesting the allocation of channel resources to an active terminal. Such a random access channel is used, among others, by cellular wireless networks, by two-way CATV networks (such as those based on the DOCSIS protocol recommendation), and by demand-assigned satellite networks. The correct design and sizing of the random access operated control/signaling channel is a critical element in determining the performance of these networks. Excessive delays in the transport of signaling messages (induced by too many collisions and retransmissions) lead to unacceptable session connection set-up times. Consequently, in this paper, we investigate the performance behavior of a random-access protocol when loaded by bursty traffic processes. The latter exhibit long range dependence (LRD). The LRD traffic flows are modeled here as multiplicative multifractal processes. The random access protocol is modeled as an Aloha channel with blocking. Parameters are defined to characterize the protocol behavior. We demonstrate that the burstiness feature of the traffic processes, rather than their LRD character, is the essential element determining the performance behavior of the protocol. When the loading traffic process is not very bursty, we show that the performance of the random-access channel can be better than that exhibited under Poisson traffic loading; otherwise, performance degradation is noted. We demonstrate the impact of the selection of the protocol operational parameters in determining the effective performance behavior of the random-access protocol.
\end{abstract}

\section{Introduction}

In the currently operating mobile, satellite, and other communications networks, Aloha-based random multiple access schemes have been extensively used especially for scenarios where the activity factor of sources is low and low packet delay is a primary requirement. Random access schemes have also been extensively used for regulating access into control (or order-wire type) channels for 
the transmission of reservation packets, as is the case for cellular wireless networks. It is essential to design such networks to yield acceptable packet delay performance to ensure that users are allocated network resources in a timely manner to satisfy their required quality of service (QoS) performance level.

The input traffic process for an Aloha-based channel is typically modeled as a Poisson process. Formulas have been developed to describe the channel's throughput and delay performance and are used for network dimensioning purposes. Recently, it has been observed that measured network traffic data often possess the long-range-dependent (LRD) property. Such traffic processes include LAN traffic [1], WAN traffic [2], variable-bit-rate (VBR) video traffic [3], and WWW traffic [4]. Furthermore it has been shown that the LRD property has a profound impact on the performance of a network. For example, Norros [5] has shown that the queue length tail distribution under the fractional Brownian motion traffic model follows an Weibull distribution instead of an exponential distribution for a queueing system under Poisson or Markovian traffic input. Erramilli et al. [6] have shown that the mean delay time for a queueing system driven by some actual LAN traffic is much worse than a queueing system driven by certain traffic processes obtained by shuffling the order of packet arrivals of the measured traffic process in such a way that the marginal distribution of the constructed traffic is the same as that of the measured traffic while the long-range-correlations are lost. Gao and Rubin [7] have shown that a Poisson model often underestimates buffer size or delay time statistics by several orders of magnitude compared to a queueing system driven by actual traffic trace data.

With LRD traffic measured in many data networks, one expects the aggregated traffic loading an Aloha-based channel to also possess such properties. Furthermore, one expects such LRD properties to have a significant impact on the performance of Aloha-based channels as well. This issue has been recently studied by Aracil and Munoz [8] and by Harpantidou and Paterakis [9]. By employing LRD real Telnet packet arrival processes as input traffic, Aracil and Munoz have found that the Aloha channel actually performs much better than that loaded by a Poisson traffic. A similar result has been obtained by Harpantidou and Paterakis, by employing a simple LRD traffic model with Pareto-distributed interarrival times as their input traffic. These results are quite the opposite of those presented by Norros [5], Erramilli et al. [6], and Gao and Rubin [7]. This situation, and the ensuing importance of the model in many applications, motivate us to carefully study the performance of Aloha channel loaded by LRD traffic.

A key issue in traffic engineering in general and LRD traffic study in particular is the characterization of the burstiness of network traffic. An LRD traffic process is characterized by the Hurst parameter $1 / 2<H<1[1,3]$. H characterizes the persistence of correlations in a traffic process and is often interpreted as an effective indicator of the burstiness of traffic [1]. Recently we have found that although an LRD traffic process is sometimes very bursty, a burstier LRD traffic is not necessarily associated with a larger value for the Hurst parameter [10]. We show here that what really matters for the performance of an Aloha 
channel is not the LRD nature, but the burstiness level, of the traffic. For this purpose, we employ a multiplicative multifractal traffic process [7,10-16] as an LRD traffic model, since multiplicative multifractal traffic processes have very well defined burstiness indicators. In this paper, we show that when the LRD traffic is not very bursty, then the performance of the random-access channel can be much better than that produced under Poisson traffic loading; otherwise, a distinct performance degradation takes place. In light of these results, we can conclude that neither the LRD Telnet arrival processes used by Aracil and Munoz [8] nor the Pareto traffic processes used by Harpantidou and Paterakis [9] are very bursty. In fact, by re-examining the latter paper, we have found that in constructing the Pareto traffic model, Harpantidou and Paterakis have deliberately selected the parameters for the Pareto traffic model in such a way that the modeled traffic becomes less and less bursty when the channel loading gets heavier.

The second purpose of this paper is to determine the desirable range for the selection of key parameter values for the random-access algorithm. This is performed to tune-up the algorithm to operate effectively under multifractal traffic, under a wide range of burstiness level conditions.

The rest of the paper is organized as follows. In Sec. 2, we describe the Aloha system adopted here and the multiplicative multifractal arrival traffic model. In Sec. 3, we first study the performance of the Aloha system under Poisson traffic, for purposes of comparing it with the same system loaded by multifractal traffic. We also examine the dependence of the performance of the system on different parameters. We then study the performance of the random-access scheme under multifractal traffic loading, assuming a wide range of burstiness levels. Finally, we draw conclusions in Sec. 4.

\section{The Multi-access Scheme and the Arrival Traffic Model}

\subsection{Slotted Aloha System with Blocking}

The system studied here is the slotted Aloha multiple-access communication channel driven by a large (theoretically infinite) number of identical users. The aggregated traffic, with mean arrival rate $\lambda$, is modeled as a multiplicative multifractal process, which will be described shortly. Each terminal handles at most a single packet at a time. All transmitted packets have the same length. A packet transmission requires a single time slot. If only one user transmits a packet in a given time slot, the packet is assumed to be correctly received by the receiver. If two or more terminals transmit packets in the same time slot, then a destructive collision occurs. For simplicity of the implementation of the system, we assume that what is detectable in a given time slot is whether the packets transmitted during the slot collide or not. The number of colliding packets is not a given observable.

The system works as follows (see the schematic of Fig. 1). A ready terminal transmits its packet at the start of a time slot. The system detects how many 
time slots experienced collisions during the most recent $W$ time slots. If, at a given time slot, this number of collisions, $N_{C S}(W)$, exceeds a threshold value, $T H$, then with probability $P_{b}$, each ready terminal is blocked during this time slot. The blocked terminal discards its packet. Non-blocked ready terminals are permitted to transmit their packets at this time slot. The latter packets are checked to confirm whether their transmissions resulted in a collision. If no collision is incurred, the transmission is declared successful. Otherwise, a collision has occurred. The collided packets are scheduled for retransmission at a later slot that is selected by using a uniform redistribution over a subsequent window of length $L$ (slots).

Next we describe the traffic model for the packet arrival process.

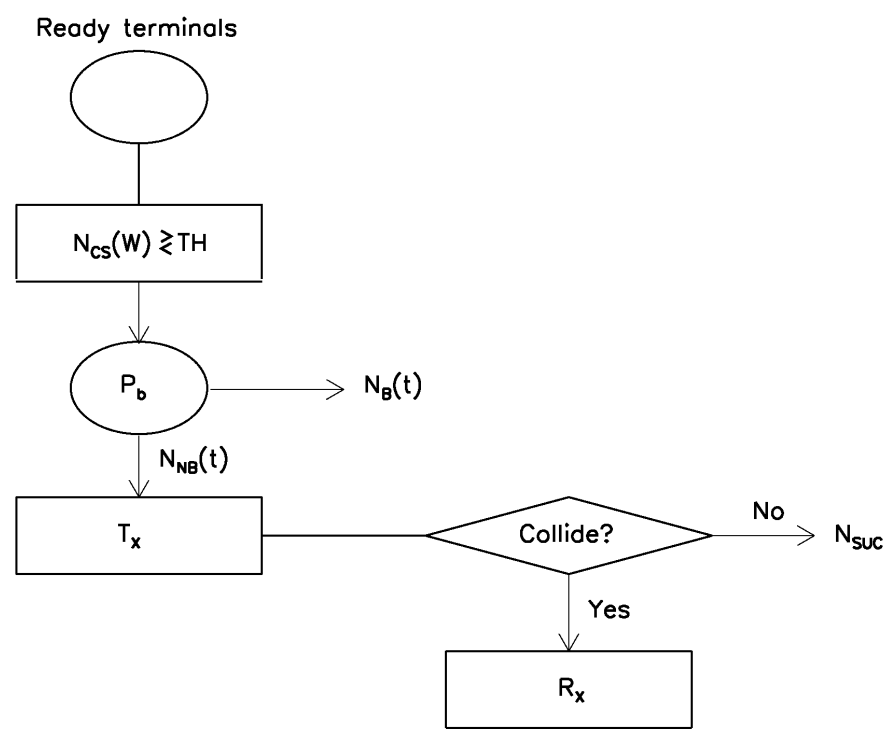

Fig. 1. Schematic of the random multi-access scheme.

\subsection{The Multiplicative Multifractal Arrival Traffic Model}

We consider the interarrival times for the aggregated traffic representing the overall arrivals of packets to the system. Note that for the Poisson traffic model, the interarrival times are assumed to be exponentially distributed. Here we employ a multiplicative multifractal traffic model, as developed by us [7, 10-16]. The model is described as follows.

Construction rule: Consider a unit interval. Associate it with a unit mass. Divide the unit interval into two (say, left and right) segments of equal length. 
Also partition the mass into two fractions, $r$ and $1-r$, and assign them to the left and right segments, respectively. The parameter $r$ is in general a random variable, governed by a probability density function $P(r), 0 \leq r \leq 1$, which is symmetric about $r=1 / 2$. The fraction $r$ is called the multiplier, and $P(r)$ is called the multiplier function. Each new subinterval and its associated weight (or mass) are further divided into two parts following the same rule (Fig. 2). The weights calculated at stage $N$ are employed to model the interarrival time series of the aggregated traffic.

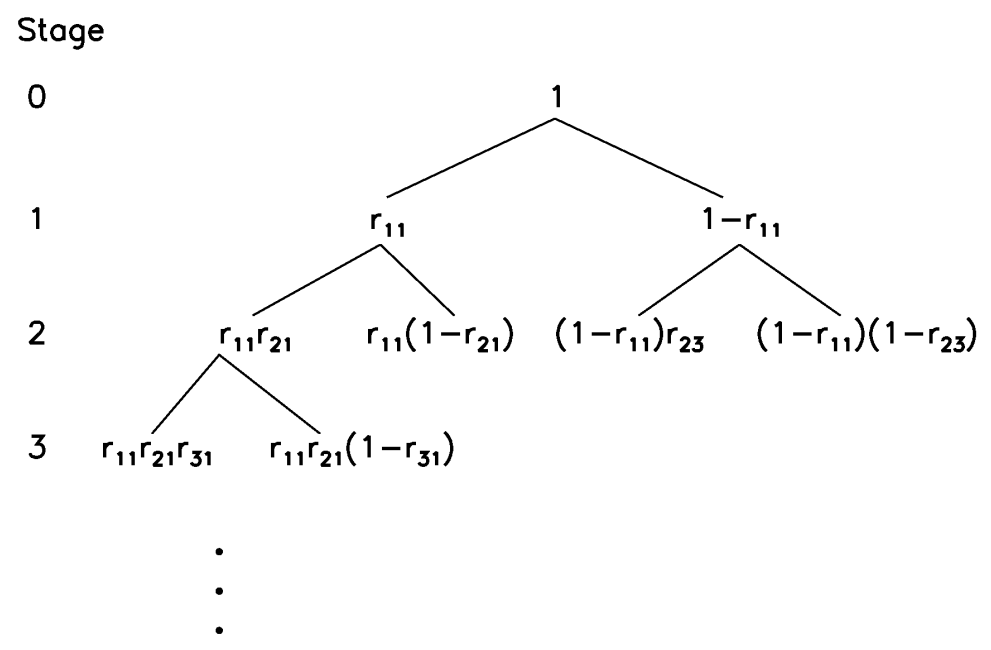

Fig. 2. Schematic of the construction rule

Among the most interesting properties of multiplicative multifractal processes are $[10,14]$ :

$-M_{q}(\epsilon)=E\left(\sum_{n=1}^{2^{N}}\left(w_{n}(N)\right)^{q}\right) \sim \epsilon^{\tau(q)}$, where $w_{n}(N)$ is a weight at stage $\mathrm{N}$, $\epsilon=2^{-N}$, and $\tau(q)=-\ln \left(2 \mu_{q}\right) / \ln 2$. This property says that multiplicative processes are multifractals.

- The weights at stage $N>>1$ have a log-normal distribution. We note that among the four different types of distributions, normal, lognormal, exponential, and Weibull distributions, the lognormal distribution is found to be the best in describing certain features of WWW traffic such as page size, page request frequency, and user's think time [17].

- Ideal multiplicative multifractals have the LTD property, with the Hurst parameter given by $1 / 2<H \leq-\frac{1}{2} \log _{2} \mu_{2}<1$.

Since $P(r)$ is symmetric about $r=1 / 2$, the mean value for the weights at stage $N$ is $2^{-N}$. In order to obtain an aggregated traffic with mean arrival rate $\lambda$, we can simply choose the length of the time slot to be $2^{-N} \lambda$. 
For simplicity, we choose the multiplier distribution to be Gaussian:

$$
P(r) \sim e^{-\alpha(r-1 / 2)^{2}} .
$$

Parameter $\alpha$ determines the burstiness of the traffic: the larger the value of $\alpha$, the less bursty the traffic is [11]. Typically, we generate a multifractal process by iterated computation till stage $N=20$, so that the number of weights at our disposal is about one million.

Since the concept of the burstiness of the traffic plays a key role in this study, we first quantitatively determine how bursty a multifractal traffic process (with given $\alpha$ ) is. For this purpose, we consider a single server FIFO queueing system with an infinite buffer. We then drive the queueing system by the multiplicative multifractal traffic and compute the queue tail size distribution. We choose the complementary queue length corresponding to the 99.99-percentile tail value as our effective measure for the burstiness of the traffic. Such a procedure can of course also be carried out for Poisson traffic. We can then normalize the 99.99 percentile of the complementary queue length for the multifractal traffic by that for Poisson traffic. Fig. 3 shows the result. We note several interesting features

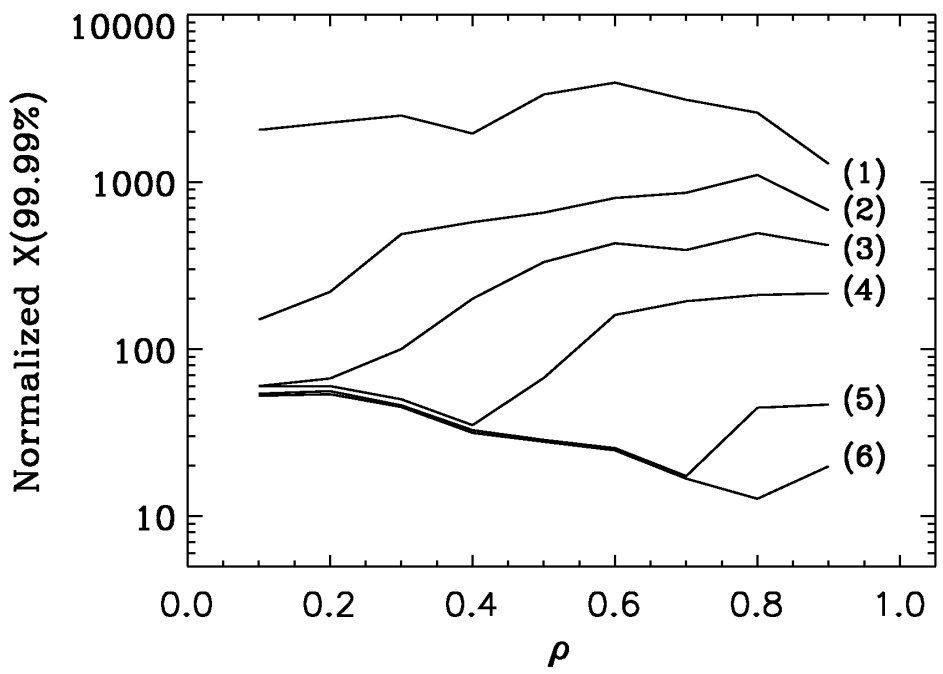

Fig. 3. The ratio of the 99.99 percentile of the complementary queue length for a FIFO queueing system with multifractal traffic and that with Poisson traffic. The six curves, denoted as (1) to (6), from top to bottom, correspond to $\alpha=10,30,50,100,400$, and 1000 .

revealed by the figure: (i) The multifractal traffic is more bursty when $\alpha$ is smaller; (ii) In terms of absolute burstiness, all the multifractal traffic processes studied here are much more bursty than the Poisson traffic; (iii) In terms of the 
rate of change of the burstiness, Poisson traffic can induce a higher such rates of change when compared to not very bursty multifractal traffic processes. This phenomenon is elucidated by the downward-trending portion of curves (4)-(6) in Fig. 3.

\section{Performance of the Aloha System}

In this section, we study the performance of the Aloha channel under different parameter values. We employ five parameters: the burstiness parameter $\alpha$; the window size $W$; the threshold value $T H$ for determining if blocking takes place; the blocking probability $P_{b}$; and the window size $L$ for redistributing the transmissions of the collided packets. For simplicity, we fix $L=10$ for the illustrative cases presented in the following. To better understand the performance of the Aloha system under multifractal traffic, we first exhibit the performance of the system under Poisson traffic. We shall only compare the best performance for the channel under Poisson traffic and that under multifractal traffic. Hence, the parameter set for the system under Poisson traffic may be different from that for the system under multifractal traffic.

\subsection{Performance of the System under Poisson Traffic}

Under the assumption that the arrival traffic is Poisson and the total aggregated traffic, which represents the superposition of new and retransmitted message process, is also Poisson, we state the well-known throughput formula:

$$
s=G e^{-G}
$$

where $s$ is the normalized throughput and $G$ denotes the total channel loading rate.

Assuming a window size of $W=20$, our simulations indicate that a good selection for the threshold level is $T H=10$. Fig. 4 shows the throughput vs. channel loading $(G)$ performance curves under three selected values for the blocking probabilities, $P_{b}=0.1,0.25$, and 0.5 . The thin solid curve is generated by using Eq. (2). We observe that all three dash-dot curves lie below the thin solid curve, indicating that the throughput of the system under Poisson arrival traffic is actually slightly lower than that predicted by Eq. (2), under the selected set of parameters (noting also that $L$ is limited to a range of 10 slots). We note that when $P_{b}$ is small, such as 0.1 , the throughput of the system becomes highly degraded as the loading rate increases. When $P_{b}$ is increased to a level such as to 0.25 , the realized throughput level is distinctly improved. However, when $P_{b}$ is further increased to a blocking level of 0.50 , the total channel loading ends at 0.7 rather than continuing to 2.0 , due to heavy blocking. Since in this case, the maximum throughput level achieved is only slightly lower than that attained for $P_{b}=0.25$, hence, this parameter value is also considered usable. As $P_{b}$ is further increased, the maximum throughput level gradually continues to decrease. 


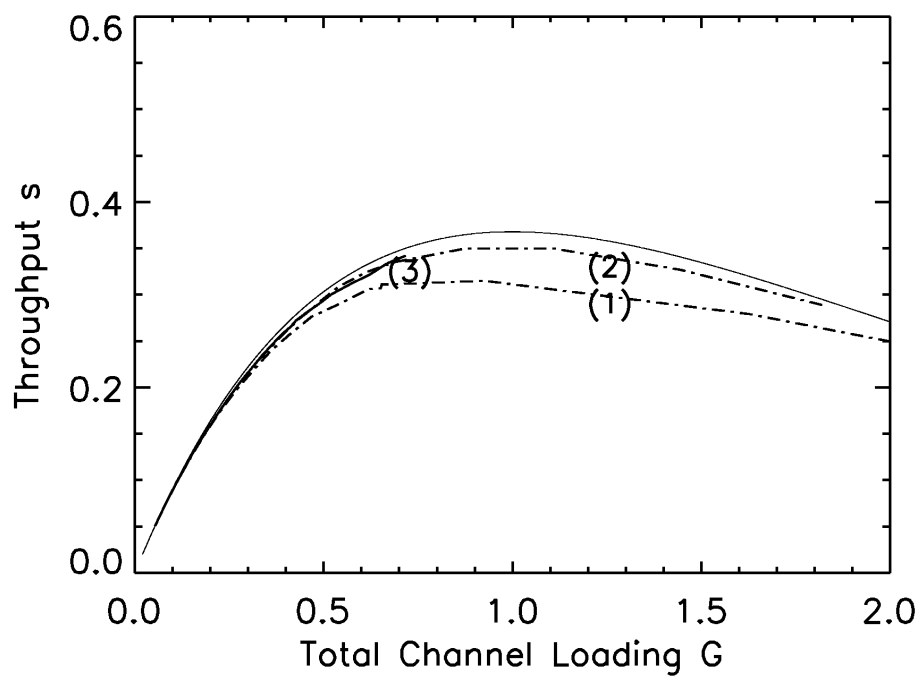

Fig. 4. The throughput $s$ vs. the total channel loading $G$ curves under Poisson traffic. The top most solid thin line is generated from Eq. (2). The other three curves, denoted as (1), (2) (both dash-dot curves), and (3) (solid thick curve), correspond to $P_{b}=0.1$, 0.25 , and 0.5 , respectively.

Packet delay time statistics also serve as important performance measures for an Aloha system. To assess the packet's delay performance, we examine the number of retransmissions incurred by a packet until it is successfully transmitted. In Fig. 5(a-c), we show the behavior of the 90-percentile of the number of retransmissions, $N_{R e t}(90 \%)$, vs. the total channel loading $G$, under Poisson traffic loading, for parameter values corresponding to the three curves exhibited in Fig. 4. It is interesting to note that the delay performance under $P_{b}=0.1$ is worse than that observed for $P_{b}=0.25$ in terms of both the realized throughput level (Fig. $4(\mathrm{a}, \mathrm{b}))$ and the attained delay performance, as expressed by $N_{R e t}(90 \%)$. For $P_{b}=0.50$, however, an improved delay performance is observed, as expected, due to the high blocking level. Under the assumed conditions, we note that designs (2) and (3) of Figs. 4-5 yield good performance behavior.

Note from Fig. 5(d), in comparison with Fig. 5(b), that as the threshold level is increased (from $T H=10$ to $T H=12$ ), message delay performance somewhat degrades. Similar throughput performance has been noted by us for the two cases (not shown for (d)).

\subsection{Performance of the System under Multifractal Traffic}

First we fix $\alpha=100$ and study the performance of the system under different parameter values for $T H, W$, and $P_{b}$. We find by numerical simulations that a good selection of $(T H, W)$ is $(7,20)$. It turns out that for not too high channel 

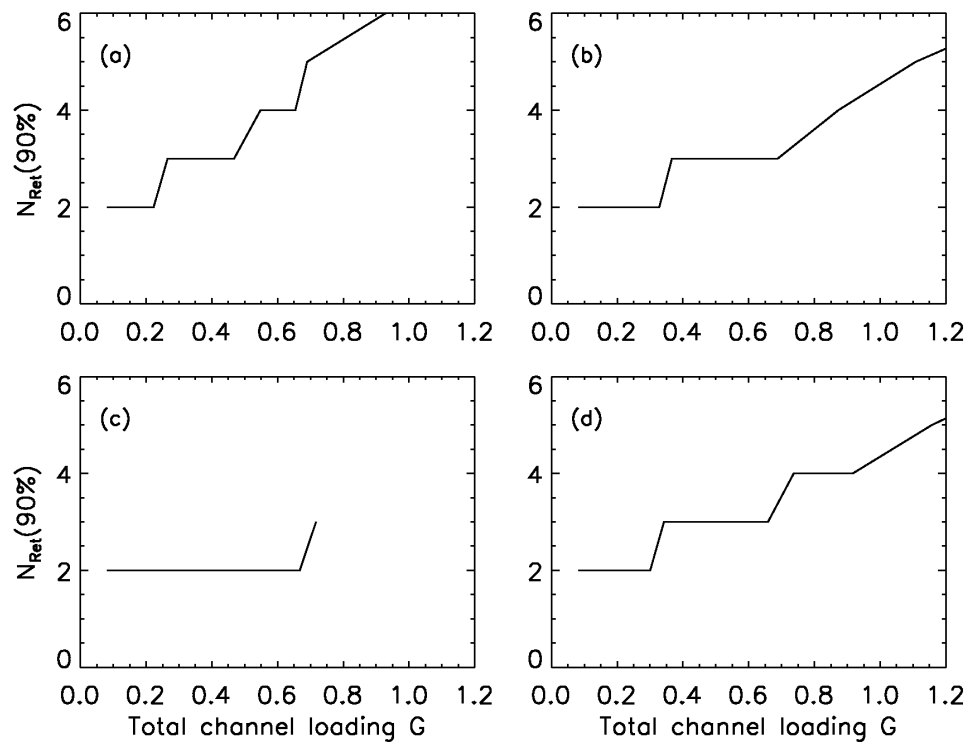

Fig. 5. The 90 percentile of the number of retransmissions vs. the total channel loading $G$ curves for Poisson traffic: (a) $P_{b}=0.1$, and $(T H, W)=(10,20)$; (b) $P_{b}=0.25$, and $(T H, W)=(10,20) ;(\mathrm{c}) P_{b}=0.5$, and $(T H, W)=(10,20)$; and $(\mathrm{d}) P_{b}=0.25$, and $(T H, W)=(12,20)$.

loading conditions, this parameter combination also works very well for the other studied $\alpha$ parameter values. Since an Aloha channel is designed to work for not too high loading conditions, we thus fix $(T H, W)$ to be $(7,20)$ in the rest of the paper.

Next, we study the dependence of the performance of the system on the blocking $P_{b}$ parameter. Fig. 6 shows the throughput $s$ vs. the total channel loading $G$ for four different $P_{b}$ values. For comparison, the curve generated from Eq. (2) is also plotted as a dashed curve. We observe that for low loading conditions, the throughput level is larger when the system is loaded by multifractal traffic (than that loaded by Poisson traffic), irrespective of the blocking probability $P_{b}$ value. Under high loading conditions, the performance of the system loaded by the LRD traffic process remains much better than that experienced under Poisson traffic loading, if suitable $P_{b}$ values ( 0.4 and 0.6 , corresponding to curves (2) and (3) in Fig. 6) are used.

It is interesting to note that a good selection of a $P_{b}$ level under multifractal traffic loading with $\alpha=100$ is higher than that used under Poisson traffic loading. When the multifractal traffic process becomes more bursty (corresponding to smaller $\alpha$ values), we find that the optimal $P_{b}$ level has to be increased gradually.

Fig. 7 shows $N_{\text {Ret }}(90 \%)$ vs. total channel loading curves for the four $P_{b}$ values studied in Fig. 6. We observe that typically when the blocking level $P_{b}$ is 


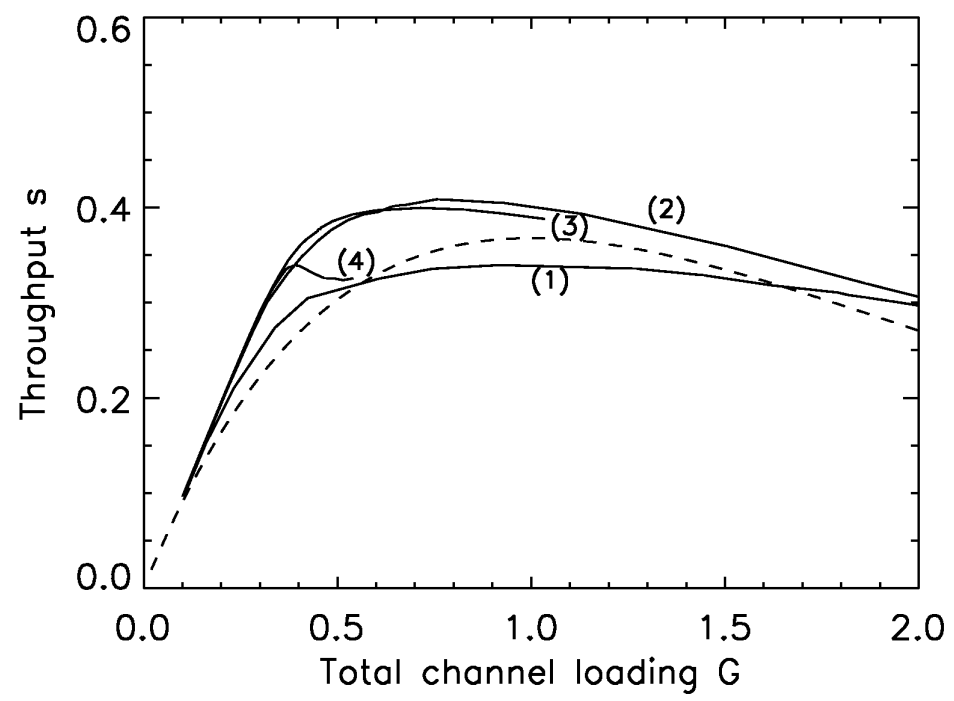

Fig. 6. The throughput $s$ vs. total channel loading $G$ curves for the Aloha system under multifractal traffic with $\alpha=100:$ (1) $P_{b}=0.2$, (2) $P_{b}=0.4$, (3) $P_{b}=0.6$, and (4) $P_{b}=0.8$.

increased, the delay performance is improved. In a satellite network, for example, $N_{\text {Ret }}(90 \%)$ is many times required to not exceed 2 (or a similarly small value, due to the high retransmission delay involved) for not too high loading conditions. Under such a criterion, a value of $P_{b}=0.6$ is most preferred. Re-examining Fig. 6, we find that this $P_{b}$ value also produces good throughput behavior.

Now that we have demonstrated the performance of the system under different values for the $T H, W$ and $P_{b}$ parameters, we study the performance of the system under multifractal traffic loading processes characterized by different burstiness levels, as expressed by their different corresponding $\alpha$ parameters. Fig. 8 shows system throughput $s$ vs. total channel loading $G$ performance curves for five different $\alpha$ levels. It is clearly noted that the attained system throughput behavior critically depends on the selected value for the loading process burstiness parameter $\alpha$ value. When the multifractal traffic is not very bursty ( $\alpha \geq 100$ ), the throughput performance of the system under multifractal traffic loading is noted to be much better than that attained under Poisson traffic loading. However, when the loading LRD traffic process becomes highly bursty, as is the case when we set $\alpha=30$ or 10, the throughput performance exhibited under multifractal traffic is much worse than that attained under Poisson traffic loading.

The delay performance for the system when loaded by multifractal traffic processes, corresponding to the conditions used for Fig. 8 (except for the case corresponding to $\alpha=100$, which is shown in Fig. $7(\mathrm{c}))$ is shown in Fig. 9. We 

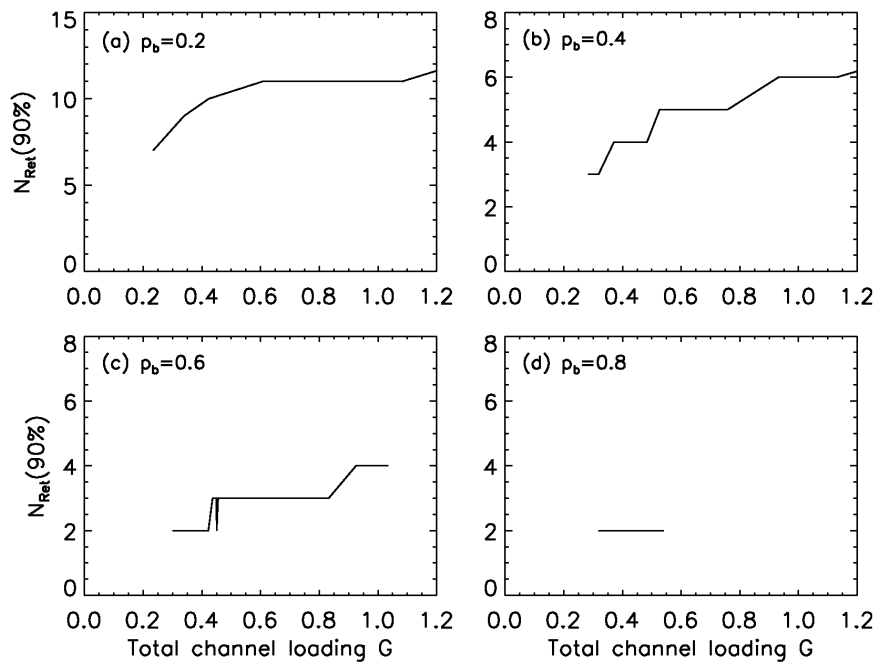

Fig. 7. The 90 percentile of the number of retransmissions vs. total channel loading $G$ curves for the system under multifractal traffic with $\alpha=100$ : (a) $P_{b}=0.2$, (b) $P_{b}=0.4$, (c) $P_{b}=0.6$, and (d) $P_{b}=0.8$.

observe that for not too high loading conditions, $N_{\text {Ret }}(90 \%)$ is less than or equal to 2 (the delay statistics for $\alpha=400$ for low loading conditions is not sufficient for the computation of $N_{\text {Ret }}(90 \%)$. In other words, such a delay requirement for $\alpha=400$ is automatically ensured).

Finally, we examine the behavior of the system's attained total blocking probability levels when the system is loaded by a multifractal traffic process, under a wide range of burstiness levels. Fig. 10 shows the corresponding blocking performance results. We observe that the blocking probability is larger when the loading process is burstier, with the blocking level attained for $\alpha=400$ being similar to that exhibited under Poisson traffic loading. Comparing curves (5) shown in Fig. 8 and Fig. 10 with corresponding curves presented by Aracil and Munoz [8], we conclude that the traffic used in [8] is much less bursty than a multifractal traffic process with $\alpha=400$.

\section{Conclusions}

In this paper, we have studied the performance of a random-access scheme when loaded by an LRD traffic loading process. The LRD traffic process is modeled as a multiplicative multifractal process. The random access scheme is the prototypical Aloha channel with blocking. Five key parameters are introduced to characterize the scheme. We show that the key performance features of the random-access protocols are determined by the burstiness level of the loading traffic process and not solely by its LRD character. We demonstrate that when the LRD loading 


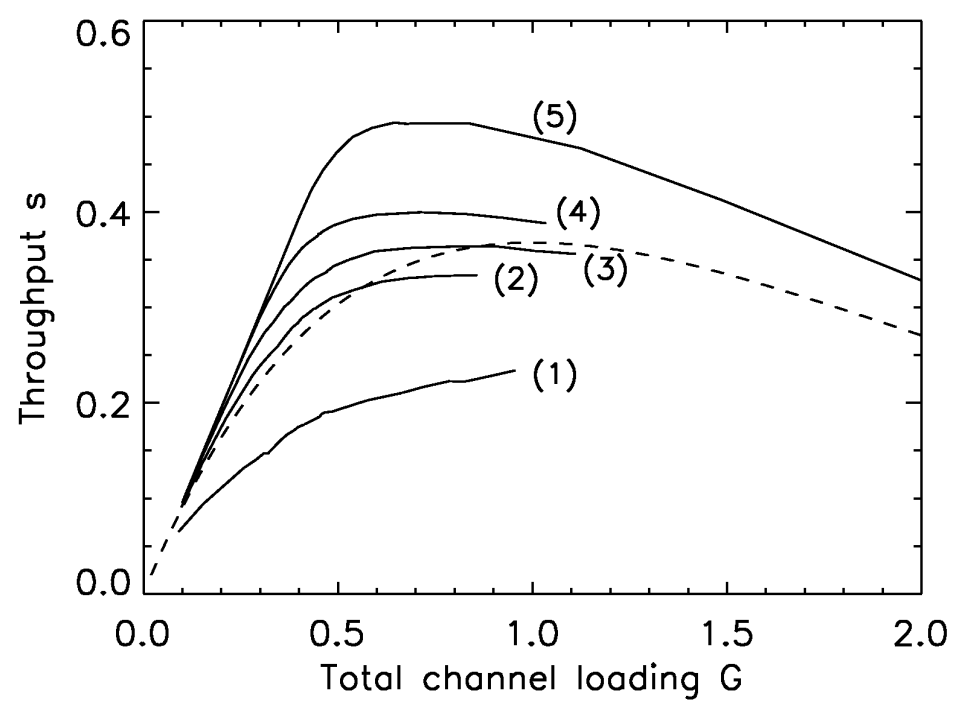

Fig. 8. Throughput $s$ vs. total channel loading $G$ curves for multifractal traffic. The 5 curves, from bottom to top, denoted as (1) to (5), correspond to $\alpha=10,30,50,100$, and 400. The dashed curve is generated from Eq. (2).
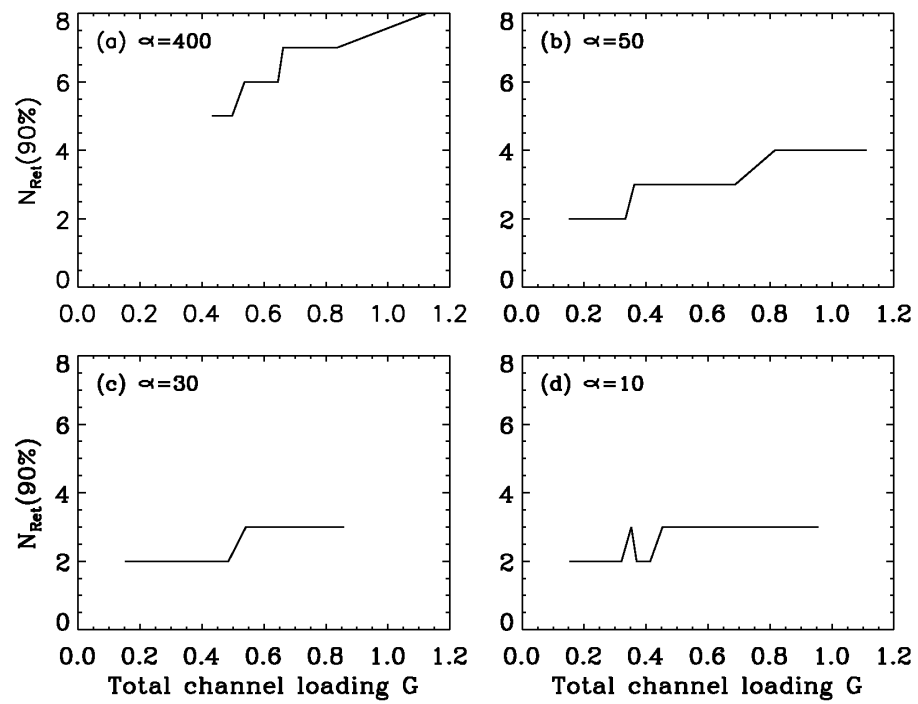

Fig. 9. The 90 percentile of the number of retransmissions vs. total channel loading $G$ curves under multifractal traffic with different $\alpha$ values. 


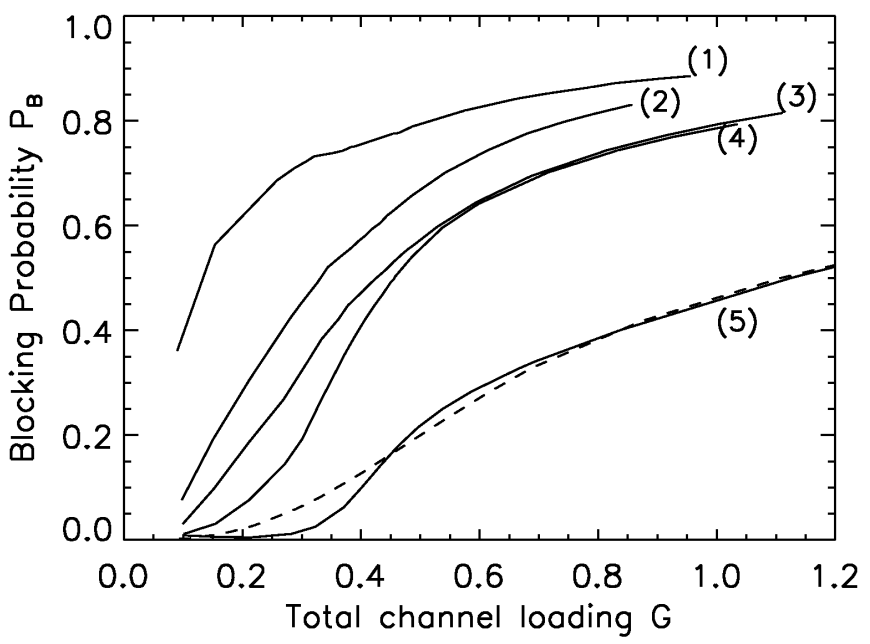

Fig. 10. Blocking probability $P_{B}$ vs. total channel loading $G$ curves. The 5 solid curves, from top to bottom, denoted as (1) to (5), correspond to multifractal traffic processes with $\alpha=10,30,50,100$, and 400. The dashed curve is for Poisson traffic loading.

process is not very bursty, the multiple-access protocol can yield better performance than that exhibited under Poisson loading conditions. In turn, significant performance degradations are observed when the system is loaded by LRD processes characterized by higher levels of burstiness. We further show that the parameters of the protocol must be tuned-up carefully to yield efficient performance behavior.

\section{References}

[1] W.E. Leland, M.S. Taqqu, W. Willinger, and D.V. Wilson, On the self-similar nature of Ethernet traffic (extended version). IEEE/ACM Trans. on Networking, 2, 1-15 (1994).

[2] V. Paxson and S. Floyd, Wide Area Traffic - The failure of Poisson modeling. IEEE/ACM Trans. on Networking, 3 226-244 (1995).

[3] J. Beran, R. Sherman, M.S. Taqqu, and W. Willinger, Long-range-dependence in variable-bit-rate video traffic. IEEE Trans. on Commun, 43 1566-1579 (1995).

[4] M.E. Crovella and A. Bestavros, Self-similarity in World Wide Web Traffic: Evidence and Possible Causes. IEEE/ACM Trans. on Networking, 5, 835-846 (1997).

[5] I. Norros, A storage model with self-similar input. Queueing Systems, 16, 387-396 (1994).

[6] A. Erramilli, O. Narayan, and W. Willinger, Experimental queueing analysis with long-range dependent packet traffic. IEEE/ACM Trans. on Networking, 4, 209223 (1996). 
[7] J.B. Gao and I. Rubin, Multiplicative Multifractal Modeling of Long-RangeDependent (LRD) Traffic in Computer Communications Networks. World Congress of Nonlinear Analysts, July, 2000, Catonia, Sicily, Italy. Also J. Nonlineae Analysis, in press.

[8] J. Aracil and L. Munoz, Performance of Aloha channels under self-similar input. Electronics Lett., 33, 659-660 (1997).

[9] Z. Harpantidou and M. Paterakis, Random multiple access of broadcast channels with pareto distributed packet interarrival times. IEEE Personal Commun., 5, 48-55 (1998).

[10] J.B. Gao and I. Rubin, Multifractal modeling of counting processes of LongRange-Dependent network Traffic. Computer Communications, in press (2001).

[11] J.B. Gao and I. Rubin, Multiplicative Multifractal Modeling of Long-RangeDependent Traffic. Proceedings ICC'99, Vancouver, Canada.

[12] J.B. Gao and I. Rubin, Multifractal modeling of counting processes of Long-Range Dependent network traffic. Proceedings SCS Advanced Simulation Technologies Conference,San Diego, CA, 1999.

[13] J.B. Gao and I. Rubin, Superposition of Multiplicative Multifractal Traffic Streams. ICC2000, June, 2000, New Orleans, Louisiana.

[14] J.B. Gao and I. Rubin, Statistical Properties of Multiplicative Multifractal Processes in Modeling Telecommunications Traffic Streams. Electronics Lett. 36, 101$102(2000)$.

[15] J.B. Gao and I. Rubin, Multifractal analysis and modeling of VBR video traffic. Electronics Lett. 36, 278-279 (2000).

[16] J.B. Gao and I. Rubin, Superposition of multiplicative multifractal traffic processes, Electronics Lett., 36: 761-762 (2000).

[17] M. Molina, P. Castelli, and G. Foddis, Web traffic modeling exploiting TCP connections' temporal clustering through HTML-REDUCE. IEEE Network, vol.14, (no.3):46-55 (2000). 